\title{
Spectral networks: a new approach to de novo discovery of protein sequences and posttranslational modifications
}

\author{
Nuno Bandeira \\ University of California, San Diego, CA, USA
}

Significant technological advances have accelerated high-throughput proteomics to the automated generation of millions of tandem mass spectra on a daily basis. In such a setup, the desire for greater sequence coverage combines with standard experimental procedures to commonly yield multiple tandem mass spectra from overlapping peptides-typical observations include peptides differing by one or two terminal amino acids and spectra from modified and unmodified variants of the same peptides. In a departure from the traditional spectrum identification algorithms that analyze each tandem mass spectrum in isolation, spectral networks define a new computational approach that instead finds and simultaneously interprets sets of spectra from overlapping peptides. In shotgun protein sequencing, spectral networks capitalize on the redundant sequence information in the aligned spectra to deliver the longest and most accurate de novo sequences ever reported for ion trap data. Also, by combining spectra from multiple modified and unmodified variants of the same peptides, spectral networks are able to bypass the dominant guess/confirm approach to the identification of posttranslational modifications and alternatively discover modifications and highly modified peptides directly from experimental data. Open-source implementations of these algorithms may be downloaded from peptide.ucsd.edu.

\section{Introduction}

Tandem mass spectrometry (MS/MS) is nowadays the technology of choice for the identification of proteins and posttranslational modifications (1). Fast-paced technological developments have delivered high-throughput analysis of thousands of proteins in a mere couple of hours at unprecedented levels of mass resolution and accuracy (2). However, the major computational approaches to the automated identification of the millions of tandem mass spectra generated on a daily basis still interpret every single tandem mass spectrum in isolation like the original techniques for de novo sequencing introduced by Klaus Biemann's group in the 1960s (3) and database searching first proposed in the early 1990s $(4,5)$. In database searching, each tandem mass spectrum is compared against a given database of known peptides and significant matches are selected for protein identification. Elaborate scoring functions have been derived to provide statistical significance to observed identifications and help make this the approach of choice for the analysis of model organisms. However, database search is only applicable when the proteins' sequences are obtained in advance through other experimental procedures, such as DNA sequencing or Edman degradation. Conversely, de novo sequencing becomes the mass spectrometric approach of choice for studies of unknown proteins. Nevertheless, fully automated de novo analysis has remained an elusive goal, due to difficulties in sequencing accuracythe best algorithms for individual ion trap tandem mass spectra still predict one incorrect amino acid out of every four predictions (6). We propose to approach the MS/MS identification problem from a different perspective-first by combining uninterpreted tandem mass spectra from overlapping peptides and only then determining consensus identifications (of sequences and modifications) for sets of aligned tandem mass spectra. Using this approach, it is possible to improve de novo sequencing accuracy to the level of one mistake out of every ten predicted amino acids and further discover many known and some putative novel modifications (7).

\section{Experimental Setup}

Most experimental protocols use enzymatic digestion to generate smaller peptides, which are then analyzed by mass spectrometry to identify proteins in the sample. Trypsin digestion is often employed because its strong cleavage specificity tends to be reproducible and facilitates the analysis of complex samples by generating only a few different peptides per protein. Alternatively, less specific enzymes or combinations of enzymes may be used to generate extensive protein coverage $(8,9)$. As illustrated in Figure 1, these procedures tend to generate many overlapping peptides covering the same protein regions. While the specificity of trypsin digestion leads to many spectra covering the same protein regions, nonspecific digestion tends to generate spectra covering large portions of the protein sequences.

After enzymatic digestion, the sample consists of a collection of peptides, usually containing sizeable amounts of most peptides. This material is then processed through a series of steps such that, in principle, each cycle of MS/ MS focuses exclusively on multiple instances of the same peptide. The same cycle is then subjected thousands of 
A

B
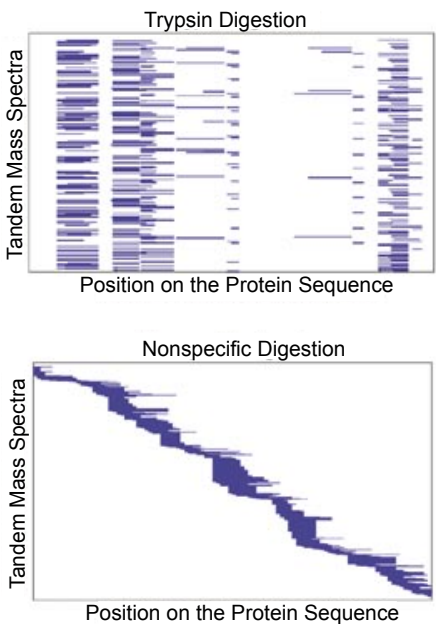

C

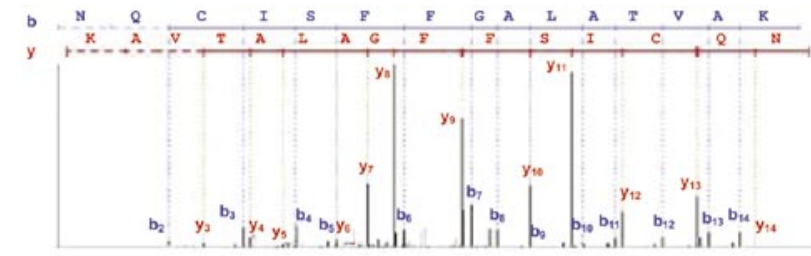

D

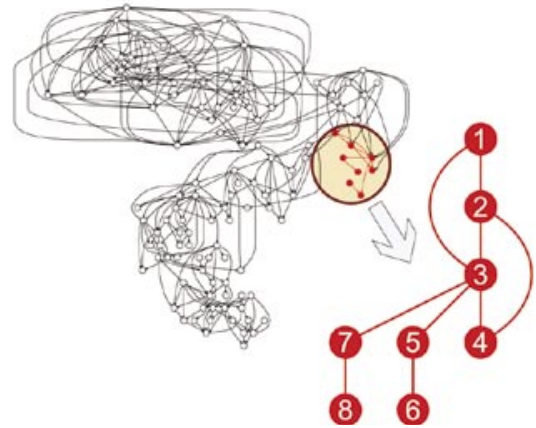

Tech Insight

Figure 1. Spectral coverage of overlapping peptides resulting from enzymatic digestion of a target protein; horizontal axes represent peptide location on the protein, and vertical axes separate different tandem mass spectra. (A) Spectral coverage resulting from trypsin digestion. (B) Spectral coverage resulting from nonspecific enzymatic digestion or digestion with multiple enzymes of different specificities. (C) Tandem mass spectrum for peptide NQCISFFGALATVAK. $b$ ions (prefix masses) are shown in blue, y ions (suffix masses) are shown in red. Note that the $b$ and y peak assignments are not known in advance but can only be determined for identified spectra. (D) Spectral network formed by a set of $117 \mathrm{IKKb}$ spectra (7). Each node corresponds to a different spectrum, and nodes are connected by an edge if the corresponding spectra were paired by spectral alignment. A subcomponent of the spectral network is shown in red along with the corresponding peptides. For example, the edge between nodes 1 and 3 indicates that the spectrum for peptide 1 was significantly aligned to the spectrum from peptide 3 .

times to a variety of procedures to maximize the number of spectra from different peptides (1). After isolating many copies of a particular peptide, a tandem mass spectrum is obtained by inducing breaks at the amide bonds, and thus generating peptide fragments whose masses and relative abundances are then measured by a mass analyzer (10). Most often, the resulting peptide fragments correspond to b ions (prefix) or y ions (suffix), although other types of ions may also be generated (see Figure $1 \mathrm{C}$ for an illustration). Since most amino acids have measurably different masses, the ion masses observed in a tandem mass spectrum typically correlate well with the theoretical masses calculated from the peptide sequence. In addition, MS/MS can be used to identify posttranslational modifications by detecting the characteristic changes in residue mass due to the addition or loss of particular compounds (11). In particular, for a modification of mass $m$, all $b$ ions and $y$ ions containing the modified residue will have their mass offset by the same mass $m$.

\section{Spectral Networks}

Samples of digested proteins often contain multiple overlapping peptides (i.e., different peptides covering the same region of a protein sequence). The simplest example is the acquisition of multiple spectra from the same peptide [sometimes detected and merged using spectral clustering techniques $(12,13)]$. However, these samples also commonly contain spectra from similar but different peptides, such as prefix peptides (e.g., PEPTI/PEPTIDES), suffix peptides (e.g., TIDES/PEPTIDES), or partially overlapping peptides (e.g., PEPTIDES/TIDESHIGH). If the peptide sequences were known in advance, determining their overlap would be a straightforward application of the standard sequence alignment algorithms (14). Conversely, spectral alignment is defined as the alignment of matching peaks between spectra from overlapping peptides $(15,16)$. This concept is illustrated in Figure $2 \mathrm{~A}$ with the matching $\mathrm{b}$ ions highlighted in blue. The surprising outcome of spectral alignment is that even though one does not know the peptide sequences in advance, it turns out that the sequence information encoded in the masses of the $b$ ions and $y$ ions is sufficient to detect pairs of tandem mass spectra from overlapping peptides.

In principle, the score of the spectral alignment between two given spectra could simply be defined as the maximum number of matched ions over all possible offsets of one spectrum in relation to the other. While this would work to a limited extent, we have found that taking into account ion intensities and correlated occurrences of multiple ion types leads to a much more accurate separation between true spectral pairs (spectra from overlapping peptides) and false spectral pairs (spurious matches between spectra from unrelated peptides). In fact, it turns out that the reliability of spectral alignment allows one to discern the high-scoring true spectral pairs from the many millions of possible spectral pairs in high-throughput proteomics experiments $(16,7)$. Moreover, since each spectrum may align to several other spectra, the set of detected spectral pairs defines a spectral network where each node corre- 


\section{Tech Insight}

sponds to a different spectrum, and nodes are connected by an edge if the corresponding spectra were found to be significantly aligned. This concept is illustrated in Figure 1D with a particular network found on a set of IKKb spectra. Note that since most spectra usually come from noncontiguous protein regions, the consequent outcome of this approach is not a single spectral network, but rather multiple spectral networks-one for each set of spectra from overlapping peptides.

\section{Shotgun Protein Sequencing}

The pattern of overlapping peptides illustrated in Figure 1B leads to particularly interesting possibilities for computational analysis-as in the assembly of genomic sequences from DNA reads, it now becomes feasible to assemble tandem mass spectra into protein sequences (17).

The assembly of spectra from overlapping peptides can be likened to a simple allegory_imagine you have a jewelry box containing many copies of a particular model of beaded necklaces. In this allegory, all necklaces are made from the same type of bead and thread, but different necklace models are characterized by designer-speci- fied varying thread distances between consecutive beads. Thus, any given necklace model is completely defined by a sequence of consecutive inter-bead distances. But what if, after collecting many copies of your favorite necklace model, you one day find that someone cut each necklace multiple times at randomly chosen bead positions? In this context, the necklace model-recovery problem is that of rediscovering the original necklace model given only the leftover pieces in the jewelry box. Although mass spectrometry adds a fair amount of complexity to this problem, this allegory captures the essence of the spectral assembly problem where amino acid masses correspond to interbead distances, and beads represent the amide bonds between consecutive amino acids.

The shotgun protein sequencing approach to de novo sequencing is a three-stage approach to the assembly of tandem mass spectra into amino acid sequences: ( $i$ ) find pairs of spectra from overlapping peptides using spectral alignment; (ii) assemble the aligned spectra; and (iii) determine a consensus amino acid sequence for each set of assembled spectra. As illustrated in Figure 2, this approach is not unlike ( $i$ ) finding necklace pieces with matching inter-bead distances; (ii) gluing the matching beads; and (iii) determining the necklace model from the recovered distances between glued beads.

By capitalizing on the correlated ion occurrences in all assembled spectra, shotgun protein sequencing leads to significant improvements in de novo sequencing accuracy and, on average, only makes one mistake out of every 10 amino acid predictions, even on low-accuracy ion trap tandem mass spectra. Using this approach, we were recently able to re-sequence large portions of multiple proteins in pure venom extract from a western

Figure 2. Shotgun protein sequencing via assembly of tandem mass spectra. (A) Spectral alignment between spectrum S1 (from peptide NMQVQWSYL) and spectrum S2 (from peptide NMQVQWSYLK) reveals the common sequence information in both spectra. Next to each spectrum is a graph representation of the corresponding peptide sequence with consecutive b ions represented as nodes connected by arrow edges. (B) Matching peaks in spectral alignments become pairwise gluing instructions between every pair of aligned spectra. Additional spectra S3 (from PQNMQVQWSYL) and S4 (from NM+16QVQWSYL), respectively, illustrate assembly of additional types of spectral alignment: partially overlapping peptides and modified/unmodified variants of the same peptide. (C) Repeated edges are replaced by single edges with weight proportional to their multiplicity and the consensus sequence for all assembled spectra is found by the heaviest path in this graph. (D) Recovered portions of a target protein in the sample. Correct amino acid predictions are shown in green (93\%) and incorrect in orange $(7 \%)$. 
Tech Insight

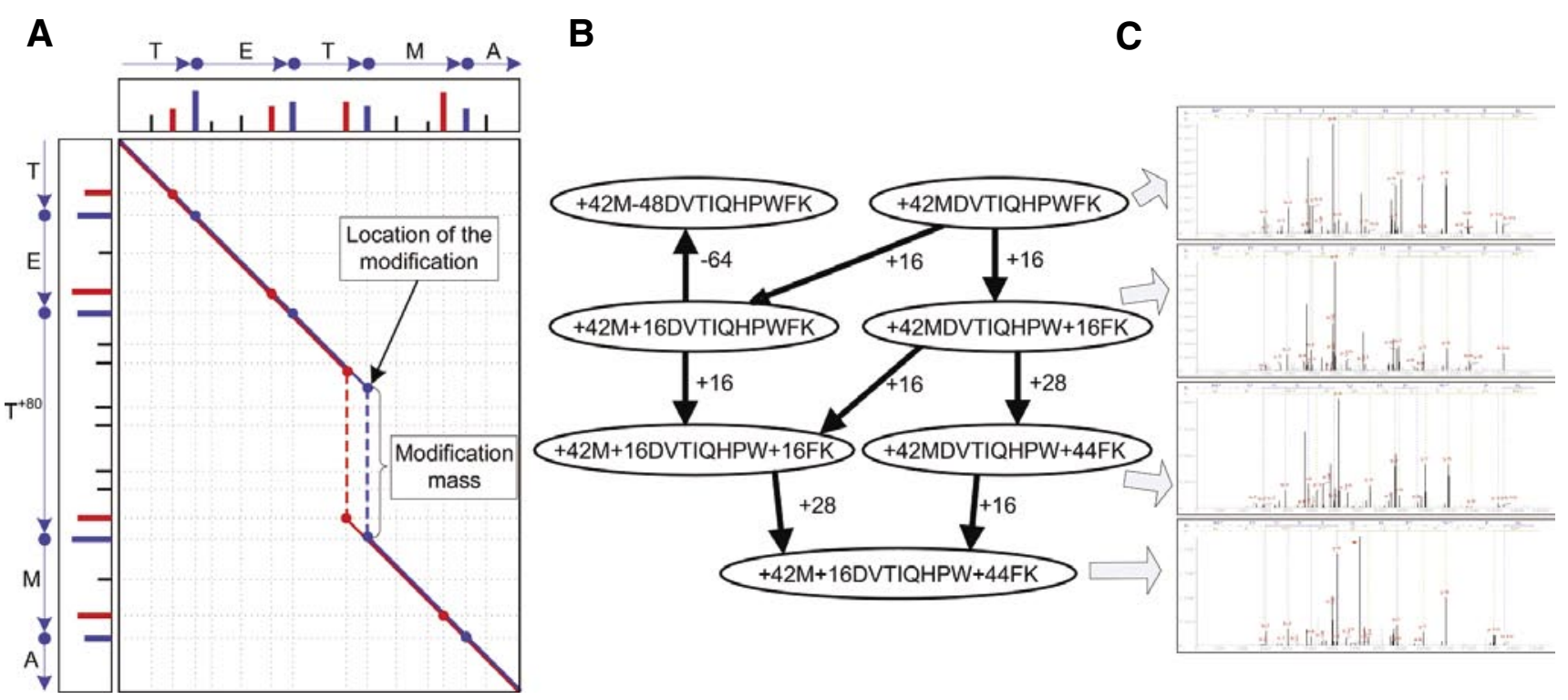

Figure 3. Identification of posttranslational modifications through spectral alignment. (A) Spectral alignment between modified and unmodified variants of the peptide TETMA ( $b$ ions shown in blue, y ions in red, blue/red lines track consecutively matched b/y ions). (B) Grouped modification states of the peptide MDVTIQHPWFK from a sample of cataractous lenses. (C) Highly correlated tandem mass spectra from the indicated peptide variants.

diamondback rattlesnake (18). In addition, compelling evidence was found for novel Crotalus atrox peptides featuring strong homology to venom peptides from other species.

\section{Spectral Networks from Spectra of Modified Peptides}

In traditional DNA sequence alignment, it often happens that query sequences differ from the reference sequences by the insertion or deletion of one or more nucleotides (14). While the insertion or deletion of amino acids is also usually allowed when aligning protein sequences, an additional factor needs to be considered when aligning peptides from experimental samples-the occurrence of posttranslational modifications. From a sequence alignment perspective, a modification could be modeled by following the modified residue with a special character for each type of modification. Thus, the alignment of a modified peptide PEPT*IDE with its unmodified counterpart PEPTIDE would result in a single difference caused by the insertion of the modification *.

Although tandem mass spectra represent peptides as a sequence of peaks, computing the spectral alignment between spectra from modified and unmodified variants of the same peptide is substantially similar to the sequence alignment problem. This correspondence can be illustrated by representing each spectrum as sequence of $1 / 0$ symbols respectively corresponding to peak/no-peak events at each mass value. Thus, for any integer mass $m$, let $s(m)$ be a sequence of $m-1$ zeros followed by a single one. For example, if an imaginary peptide of mass 12 was composed by amino acids XYZ (with masses 3,4,5, respectively), then its theoretical spectrum would contain peaks at masses 3,7,12, and the corresponding 0/1-sequence representation would be s(3)s(4)s(5) $=001000100001$. In this framework, any sequence of masses (such as a peptide or a modified peptide) can be expressed as a sequence of $0 / 1$ symbols, and pairs of sequences can then be aligned using standard sequence alignment algorithms (14). As such, a modification of mass $m^{\prime}$ corresponds to the insertion of $m^{\prime}$ additional zeros right before the sequence for the modified residue (i.e., the mass of the residue becomes larger). Conversely, if the modification causes a loss of $m^{\prime \prime}$ Daltons (mass units) from the modified residue, then the corresponding effect is the deletion of $m^{\prime \prime}$ zeros from the sequence for the modified residue. Although spectral alignment algorithms do not explicitly convert spectra to sequences of zeros and ones, this model illustrates the essential concepts behind the approach. Figure 3A illustrates the spectral alignment between tandem mass spectra from the peptides TETMA and TET ${ }^{+80} \mathrm{MA}$. When first analyzing a sample possibly containing modified peptides, one does not know a priori which residues or peptides will be modified. Thus, spectral alignment considers every possible spectral pair and every possible location for the mass difference (e.g., modification mass) between the aligned spectra. By requiring a significant match between the aligned spectrum peaks (7), but placing no restrictions on which modifications to consider, this approach can be used to discover novel or unexpected modifications. In fact, when applied to a set of spectra from cataractous lens proteins from a 93-year-old patient, spectral networks were able to rediscover the modifications identified by database search methods and additionally discovered several novel modification events $(19,7)$.

The identification of peptides containing multiple modi- 
fications via database search is a challenging problem because of the combinatorial explosion in the number of possible modification variants for all the peptides in a database (19). Not only can the large number of possible peptide variants make this approach much slower, but the increased number of peptide candidates for any given spectrum significantly increases the risk of incorrect identifications. However, samples containing peptides with two or more modifications often also contain variants of the same peptide with only one or no modification. In these cases, we have found that spectral alignment is able to group these related spectra from multiple modification variants of the same peptide into small spectral networks. Figure 3B illustrates the spectral network for a particular peptide in a sample of cataractous lens proteins.

By grouping together spectra from multiple variants of the same peptide, spectral networks additionally contribute to the reliable identification of highly modified peptides. While database searching is restricted to matching ion masses between theoretical and observed spectra, spectral networks further capitalize on the correlated co-occurrences of ions at corresponding masses and with similar peak intensities (Figure 3C). In general terms, it becomes easier to identify a highly modified peptide if one additionally observes highly similar spectra from the intermediate modification states. Thus, spectral alignment not only allows one to discover unexpected modifications (instead of only identifying expected modifications) but additionally defines an alternative way to reliably identify highly modified peptides.

\section{Conclusion}

Spectra from overlapping peptides or modification variants of the same peptide deliver a wealth of correlated sequence information that can be explored with a new generation of algorithms based on spectral networks. In a departure from standard procedures, having spectra from modified or unmodified variants of the same peptide allows one to directly discover the modifications in the sample rather than having to guess in advance the list of modifications to search for. Spectra from multiple modification variants can be combined into spectral networks and correlated ion masses and intensities used to increase the confidence in the identification of highly modified peptides. From a protein sequencing perspective, the extensive sequence coverage achievable with nonspecific proteolytic digestion enables the assembly of spectra from overlapping peptides into long protein contigs. Moreover, by capitalizing on the correlated sequence information in sets of assembled spectra, the shotgun protein sequencing approach is able to deliver the highest sequencing accuracy ever reported on ion trap tandem mass spectra.

\section{References}

1. Aebersold, R. and M. Mann. 2003. Mass spectrometry-based proteomics. Nature 422:198-207.
2. Yates, J.R. 2004. Mass spectrometry as an emerging tool for systems biology. BioTechniques 36:917-919.

3. Biemann, K., C. Cone, B.R. Webster, and G.P. Arsenault. 1966. Determination of the amino acid sequence in oligopeptides by computer interpretation of their high-resolution mass spectra. J. Am. Chem. Soc. 88:5598-5606.

4. Henzel, W.J., T.M. Billeci, J.T. Stults, S.C. Wong, C. Grimley, and C. Watanabe. 1993. Identifying proteins from two-dimensional gels by molecular mass searching of peptide fragments in protein sequence databases. Proc. Natl. Acad. Sci. USA 90:5011-5015.

5. Yates, J.R., J.K. Eng, and A.L. McCormack. 1995. Mining genomes: correlating tandem mass spectra of modified and unmodified peptides to sequences in nucleotide databases. Anal. Chem. 67:3202-3210.

6. Fischer, B., V. Roth, F. Roos, J. Grossmann, S. Baginsky, P. Widmayer, W. Gruissem, and J.M. Buhmann. 2005. Novohmm: a hidden markov model for de novo peptide sequencing. Anal. Chem. 77:7265-7273.

7. Bandeira, N., D. Tsur, A. Frank, and P.A. Pevzner. 2007. Protein identification via spectral networks analysis. Proc. Natl. Acad. Sci. USA 104:6140-6145.

8. MacCoss, M.J., W.H. McDonald, A. Saraf, R. Sadygov, J.M. Clark, J.J. Tasto, K.L. Gould, D. Wolters, et al. 2002. Shotgun identification of protein modifications from protein complexes and lens tissue. Proc. Natl. Acad. Sci. USA 99:7900-7905.

9. Englander, J.J., C. Del Mar, W. Li, S.W. Englander, J.S. Kim, D.D. Stranz, Y. Hamuro, and V.L. Woods. 2003. Protein structure change studied by hydrogen-deuterium exchange, functional labeling, and mass spectrometry. Proc. Natl. Acad. Sci. USA 100:7057-7062.

10.Siuzdak, G. 2003. Mass Spectrometry in Biotechnology. MCC Press, San Diego.

11. Jensen, O.N. 2006. Interpreting the protein language using proteomics. Nat. Rev. Mol. Cell Biol. 7:391-403.

12. Tabb, D.L., M.J. MacCoss, C.C. Wu, S.D. Anderson, and J.R. Yates, 3rd. 2003. Similarity among tandem mass spectra from proteomic experiments: detection, significance, and utility. Anal. Chem. 75:2470-2477.

13. Beer, I., E. Barnea, T. Ziv, and A. Admon. 2004. Improving large-scale proteomics by clustering of mass spectrometry data. Proteomics 4:950-960.

14. Smith, T.F. and M.S. Waterman. 1981. Identification of common molecular subsequences. J. Mol. Biol. 147:195-197.

15. Pevzner, P.A., V. Dancík, and C.L. Tang. 2000. Mutation-tolerant protein identification by mass spectrometry. J. Comput. Biol. 7:777-787.

16. Bandeira, N., D. Tsur, A. Frank, and P.A. Pevzner. 2006. A new approach to protein identification, p. 363-378. In A. Apostolico, C. Guerra, S. Istrail, P.A. Pevzner, and M. Waterman (Eds.), Lecture Notes in Computer Science 3909: Proceeding of the Tenth Annual International Conference in Research in Computational Molecular Biology. Springer, New York.

17. Bandeira, N., H. Tang, V. Bafna, and P. Pevzner. 2004. Shotgun protein sequencing by tandem mass spectra assembly. Anal. Chem. 76:7221-7233.

18. Bandeira, N., K. Clauser, and P.A. Pevzner. Shotgun protein sequencing: assembly of tandem mass spectra from mixtures of modified proteins. Mol Cell Proteomics (in press).

19. Tsur, D., S. Tanner, E. Zandi, V. Bafna, and P.A. Pevzner. 2005. Identification of post-translational modifications by blind search of mass spectra. Nat. Biotechnol. 23:1562-1567. 